\title{
Hemianopsia por lesão no corpo geniculado lateral. Importância diagnóstica da análise da camada de fibras nervosas pela tomografia por coerência óptica: relato de caso
}

\author{
Geniculatehemianopia. Diagnostic importance of retinal nerve fiberlayer analysis \\ usingoptical coherence tomography:case report
}

\author{
Frederico Castelo Moura ${ }^{1}$ \\ Patrícia Lunardelli ${ }^{2}$ \\ Cláudia Costa Leite ${ }^{3}$ \\ Mário Luiz Ribeiro Monteiro ${ }^{4}$
}

\begin{tabular}{|l|}
\hline RESUMO \\
\hline Lesões que acometem o corpo geniculado lateral (CGL) são as mais raras \\
entre as afecções da via óptica. Os exames de neuro-imagem têm a \\
importante função de identificar tais lesões. Entretanto, devido a seus \\
tamanho e localização, a demonstração neuro-radiológica do corpo geni- \\
culado lateral e de suas lesões, podem eventualmente, ser dificultadas \\
levando a confusão diagnóstica. O objetivo desse relato é documentar um \\
caso raro de acometimento do corpo geniculado lateral e em que tomografia \\
por coerência óptica teve participação relevante na confirmação do diag- \\
nóstico anatômico de lesão do corpo geniculado lateral. Paciente feminina \\
de 39 anos, com diagnóstico prévio de uveíte e vasculite de sistema \\
nervoso central por doença de Behçet, apresentou-se para a investigação \\
de quadrantopsia temporal à direita. A imagem por ressonância magnética \\
não mostrava lesão na via óptica. Evidenciou-se à oftalmoscopia,perda da \\
camada de fibras nervosas retinianas peripapilar "em banda", que foi \\
confirmada pela tomografia porcoerênciaóptica. Esta observação, associada \\
aos achados do exame neurológico levaram à forte suspeita de lesão no \\
corpo geniculado lateral sendo então solicitada nova imagem por resso- \\
nância magnética, que evidenciou lesão isquêmica de corpo geniculado \\
lateral. O caso apresentado confirma a importância do tomógrafo por \\
coerência óptica em determinar o padrão de perda de camada de fibras \\
nervosas retinianas em afecções neuroftálmicas, e assim cooperar na \\
localização da lesão dentro da via óptica.
\end{tabular}

Descritores: Corpos geniculados; Hemianopsia; Células do gânglio retiniano; Fibras nervosas; Doenças do nervo óptico/patologia; Tomografia de coerência óptica/métodos; Condutos visuais; Relatos de casos [tipo de publicação]

\footnotetext{
Pós-graduando, nível doutorado da Divisão de Clínica Oftalmológica do Hospital das Clínicas da Faculdade de Medicina da Universidade de São Paulo (USP). São Paulo (SP)

Residente do $2^{\circ}$ ano da Divisão de Clínica Oftalmológica do Hospital das Clínicas da Faculdade de Medicina da USP. São Paulo (SP).

${ }^{3}$ Residente do $2^{\circ}$. Ano do Departamento de Radiologia do Hospital das Clínicas da Faculdade de Medicina da USP. São Paulo (SP)

${ }^{4}$ Professor Livre Docente da Divisão Oftalmológica do Hospital das Clínicas da Faculdade de Medicina da USP. São Paulo (SP).

Endereço para correspondência: Mário Luiz Ribeiro Monteiro - Av. Angélica 1757 - Conj. 61 - São Paulo (SP) CEP 01227-200

E-mail: mlrmonteiro@terra.com.br

Recebemos para publicação em 05.07.2004

Aprovação em 15.12.2004

Nota Editorial: Depois de concluída a análise do artigo sob sigilo editorial e com a anuência do Dr. Marco Aurélio Lana Peixoto sobre a divulgação de seu nome como revisor, agradecemos sua participação neste processo.
}

\section{INTRODUCÃO}

Lesões que acometem o corpo geniculado lateral (CGL) são as mais raras entre as afecções da via óptica. A suspeita dessas lesões baseia-se no tipo de defeito campimétrico apresentado associado ao padrão de perda da camada de fibras nervosas retinianas (CFNR) observado à oftalmoscopia, aos achados no exame das pupilas e aos achados neurológicos associa$\operatorname{dos}^{(1)}$. O estudo de imagem pela tomografia computadorizada ou pela ressonância magnética tem a importante função de localizar a lesão e confirmar o diagnóstico. Entretanto, quando os achados aos exames de imagem são 
discretos, tais como ocorre em pequenas lesões isquêmicas do $\mathrm{CGL}^{(2)}$ o diagnóstico pode ser difícil e depender fundamentalmente dos achados clínicos e do defeito campimétrico.

Métodos que avaliam, de uma forma objetiva, a camada de fibras nervosas retinianas, determinam o padrão de perda da mesma, e assim podem auxiliar na localização de lesões que acometem a via óptica. A tomografia por coerência óptica é um dos métodos que apresentam essa finalidade por avaliar a CFNR peripapilar em todos os setores, entre eles, os setores nasal e temporal que apresentam uma importância especial no estudo das afecções neuroftalmológicas ${ }^{(3)}$.

Apresentamos nesse trabalho um caso de hemianopsia geniculada causado por vasculite decorrente de doença de Behçet, onde a tomografia por coerência óptica teve participação relevante na confirmação do diagnóstico anatômico da lesão de CGL.

\section{RELATO DE CASO}

Paciente do sexo feminino, 39 anos, parda, com diagnóstico prévio de vasculite de sistema nervoso central por doença de Behçet foi encaminhada ao serviço de Neuroftalmologia da Faculdade de Medicina da Universidade de São Paulo - FMUSP por apresentar, em olho direito, quadrantopsia temporal com a mira $\mathrm{V} / 4 \mathrm{e}$ e hemianopsia temporal com as demais miras (Figura 1), sem uma lesão neuro-radiológica que explicasse tal achado campimétrico. Em princípio, considerou-se que o defeito de campo visual era devido a glaucoma, uma vez que os exames de imagem não demonstravam anormalidade na via óptica que pudesse explicar tal defeito. A paciente referia diagnóstico de acometimento neurológico por doença de Behçet que foi esta-

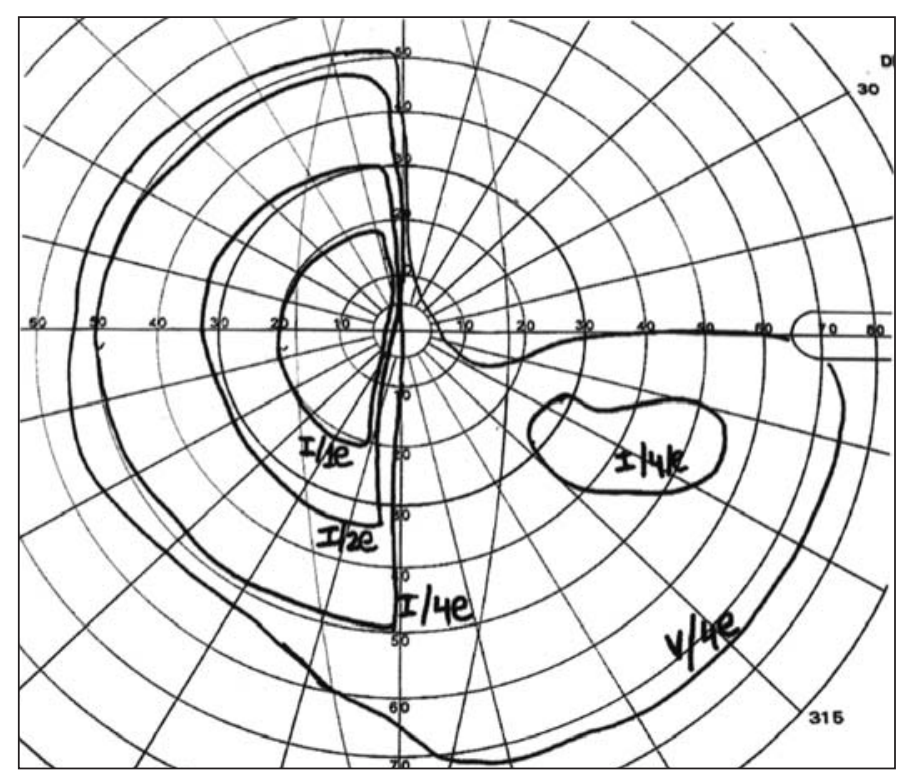

Figura 1 - Campo visual do olho direito evidenciando quadrantopsia temporal superior com mira $\mathrm{V} / 4 \mathrm{e}$ e hemianopsia temporal com as demais miras belecido quando apresentou quadro de hemiparesia à direita, uveíte anterior em olho esquerdo e úlceras orais recorrentes há dez anos. Referia que naquela época, além do acometimento visual no olho esquerdo (OE), apresentou perda do campo visual no olho direito (OD) que permaneceu inalterado apesar da melhora dos sintomas neurológicos após o tratamento.

Ao exame oftalmológico, apresentava acuidade visual de 20/20 no OD e ausência de percepção luminosa no OE que se apresentava em "phitisis bulbi" decorrente de uveíte hipertensiva prévia. Biomicroscopia do segmento anterior, motilidade ocular extrínseca e reflexo pupilar direto no olho direito se apresentavam sem alterações. A fundoscopia do olho direito revelou palidez temporal de papila e aspecto sugestivo de perda da CFNR peripapilar "em banda" neste olho (Figura 2). $\mathrm{O}$ exame de imagem por ressonância magnética inicialmente não possibilitou a identificação de qualquer lesão que acometesse a via óptica especialmente na região quiasmática e retroquiasmática. O resultado deste exame de imagem por ressonância magnética levou à dúvida diagnóstica quanto ao possível local da lesão ao longo da via óptica. O fato de o paciente ter apenas um dos olhos com visão não permitiu a análise de possível congruência ou não deste defeito. Devido a suspeita de acometimento da CFNR observada à oftalmoscopia foi realizada tomografia por coerência óptica, utilizando o modelo comercial STRATUS ${ }^{\text {TM }}$ OCT (Carl Zeiss Meditec Inc., Dublin, CA, USA), que revelou perda da camada de fibras nervosas retinianas peripapilar, preferencialmente, nos setores temporal e nasal do disco óptico do olho direito confirmando a suspeita de atrofia em banda da CFNR (Figura 3).

Após a confirmação da perda da CFNR, concluiu-se por uma lesão que acometesse a via óptica anterior ao CGL, sendo esta localização a mais provável tendo em vista o dado de história de uma hemiparesia associada à perda da visão lateral quando a paciente apresentou vasculite do sistema nervoso central. Frente à possibilidade da lesão na via óptica estar localizada no CGL, solicitou-se um novo exame de ressonância

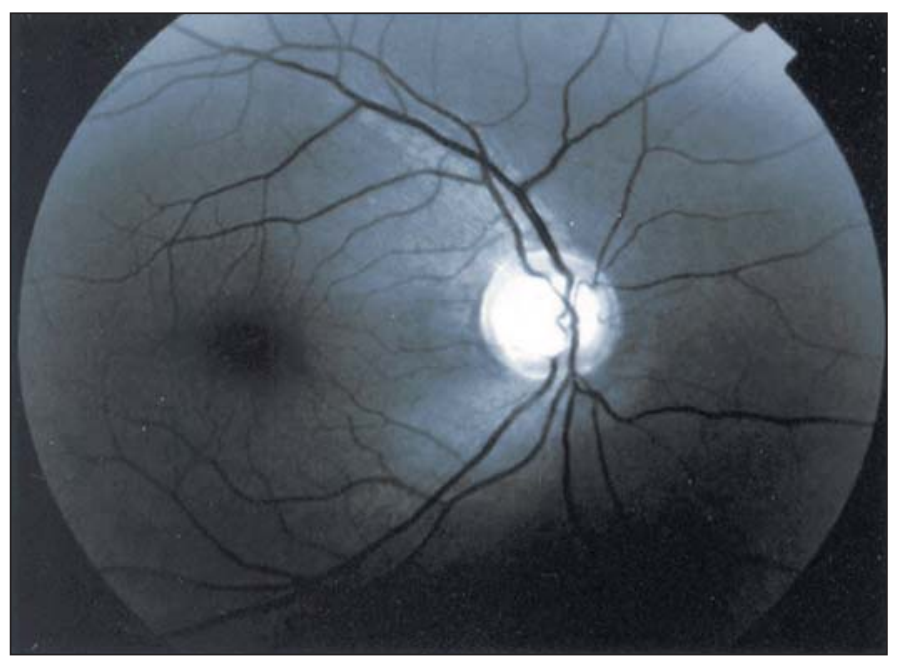

Figura 2 - Retinografia com luz aneritra do olho direito evidenciando perda da camada de fibras nervosas nos setores nasal e temporal 


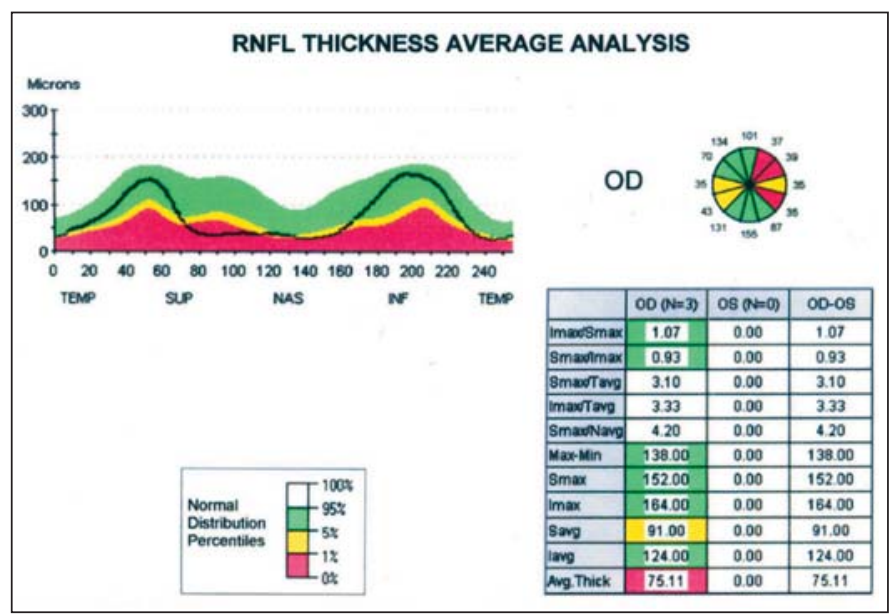

Figura 3 - Análise da camada de fibras nervosas retiniana pela tomografia por coerência óptica mostrando redução das fibras, especialmente, nos setores nasal e temporal

magnética apropriado ao estudo dessa região. O resultado confirmou a presença de uma lesão isquêmica acometendo o CGL à esquerda (Figura 4) permitindo estabelecer a lesão causal da quadrantopsia.

\section{DISCUSSÃO}

Lesões isoladas do CGL são raras entre as afecções da via óptica, provavelmente, devido a sua peculiar localização. A identificação dessas lesões é fundamentada pelo padrão de perda da camada de fibras nervosas retinianas, ou seja, atrofia em banda no olho contralateral e atrofia difusa no olho ipsilateral (semelhante ao padrão de lesão do trato óptico) ${ }^{(4)}$ e os achados à campimetria, que podem apresentar-se como hemianopsia homônima ${ }^{(5)}$, setoranopsia horizontal homônima ${ }^{(6)}$, setoranopsia quádrupla homônima ${ }^{(7)}$ e defeito em "ampulheta" em ambos os campos visuais ("hourglass-shaped defect"), este último ocorrendo em acometimento bilateral do $\mathrm{CGL}^{(8)}$.

$\mathrm{O}$ estudo neuro-radiológico corrobora na confirmação do
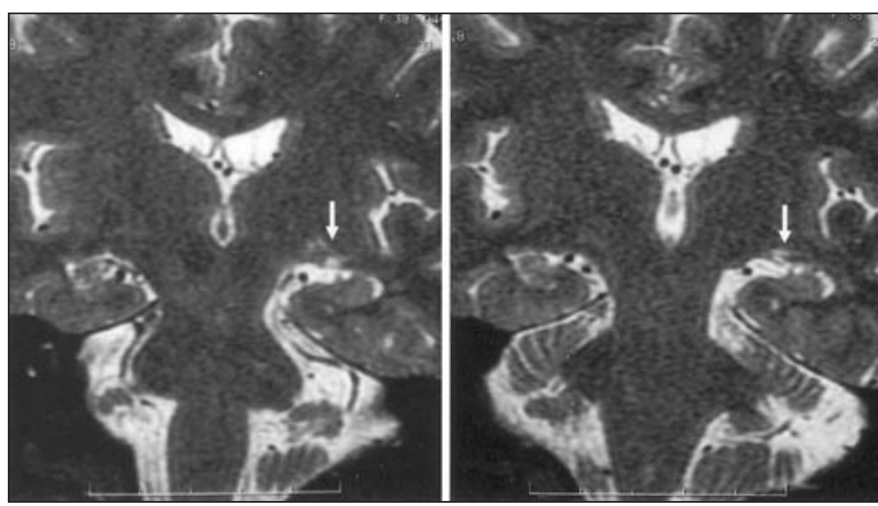

Figura 4 - Imagens por ressonância magnética (em T2) em 2 cortes coronais seqüenciais mostrando aumento da intensidade do sinal na região correspondente ao corpo geniculado lateral esquerdo (setas) diagnóstico anatômico das afecções da via óptica. Nos relatos prévios de lesão de CGL, os métodos de imagem, tanto tomografia computadorizada como ressonância magnética, foram importantes na localização e identificação das afecções envolvidas, entre elas, infartos ${ }^{(9)}$, tumores ${ }^{(10)}$, mielinose extra-pontina central ${ }^{(8)}$ e trauma ${ }^{(11)}$. Todavia, devido a seus tamanho e localização, a demonstração neuro-radiológica do CGL e de suas lesões podem, eventualmente, ser dificultadas, como ocorre, por exemplo, em lesões isquêmicas ${ }^{(2)}$.

A possibilidade da lesão na via óptica em questão estar localizada no CGL esquerdo foi sugerida e fundamentada pelo quadro de hemiparesia à direita (devido ao comprometimento do trato piramidal esquerdo próximo ao CGL ipsilateral) ${ }^{(7)}$, pelo defeito hemianóptico temporal à direita e pela perda da CFNR nos setores nasal e temporal visualizada à oftalmoscopia e confirmada por tomografia de coerência óptica. No paciente em estudo, a ausência de visão em um dos olhos por "phitisis bulbi" representou uma dificuldade diagnóstica adicional, pois impedia a avaliação da presença ou não de defeito pupilar aferente e, também, de avaliar a simetria ou não do defeito campimétrico de um olho com o outro. Nos pacientes que apresentam visão nos dois olhos, a ausência de defeito pupilar aferente relativo no olho com a hemianopsia temporal serve como um dado a mais no diagnóstico de lesão no CGL e auxilia na diferenciação com as lesões do trato óptico (que usualmente se acompanham de defeito pupilar aferente relativo).

Diante da possibilidade de lesão do CGL, o exame de imagem por ressonância magnética foi repetido utilizando-se parâmetros técnicos especiais que facilitaram a identificação do CGL. Esses parâmetros foram baseados em um estudo anátomo-radiológico ${ }^{(12)}$ onde os autores compararam as imagens por ressonância magnética do CGL de peças anatômicas cerebrais obtidas em autópsia com imagens da mesma região de pacientes vivos normais. Atenção especial foi dada à região $\operatorname{logo}$ acima do recesso lateral da cisterna ambiens, em frente ao subiculum da formação parahipocampal, onde o CGL é descrito em atlas de anatomia cerebral. Para isso, foram obtidas imagens ao nível do tálamo em um plano coronal levemente inclinado para frente e paralelo ao soalho do quarto ventrículo. O resultado desse novo exame confirmou nossa suspeita de lesão isquêmica do CGL à esquerda e serviu para esclarecer definitivamente a causa do defeito campimétrico. Embora a suspeita clínica tenha sido o fator decisivo para o diagnóstico, a informação obtida através da tomografia por coerência óptica revelou-se de importância fundamental dando suporte aos achados clínicos e reforçando a suspeita diagnóstica. O caso apresentado confirma a importância da tomografia por coerência óptica no estudo das afecções neuroftalmológicas por avaliar, de uma forma objetiva, a CFNR peripapilar, identificar o padrão de perda da mesma e, assim, cooperar com a localização da lesão dentro da via óptica. Este caso serve também para enfatizar a importância da localização precisa de uma lesão ao longo da via óptica de tal forma a possibilitar ao neurorradiologista a realização de um exame com ênfase ao local da suspeita diagnóstica o que pode facilitar muito o estabelecimento do diagnóstico correto. 


\section{ABSTRACT}

Lesions of the lateral geniculate body (LGB) are the most unusual lesions of the visual pathways. Imaging studies are very important in establishing the correct diagnosis. However, due to its small size and particular location, the lateral geniculate body and its lesions are sometimes difficult to detect in imaging studies possibly causing diagnostic confusion. The purpose of this paper is to document an unusual case of a lesion of the lateral geniculate body for which an optical coherence tomography study was very important in confirming the anatomic diagnosis of a lateral geniculate body lesion. A 39-year-old woman with a previous diagnosis of uveitis and central nervous system vasculitis was referred for investigation of a right temporal quadrantanopia. She had already been submitted to a magnetic resonance imaging (MRI) that did not show any lesion along the visual pathway. Ophthalmoscopy revealed retinal nerve fiber layer (RNFL) loss that was confirmed by optical coherence tomography. Such finding associated with the observations on the neurological examination strongly suggested a lateral geniculate body lesion. The patient was submitted to another new magnetic resonance imaging obtained with especially oriented thin sections and an ischemic lesion of the lateral geniculate body was observed establishing the correct diagnosis. This case serves to confirm the importance of optical coherence tomography in determining the pattern of retinal nerve fiber layer loss in neuro-ophthalmic diseases and therefore to help in locating a lesion along the visual pathway.
Keywords: Geniculate bodies; Hemianopsia; Retinal ganglion cells; Nerve fibers/pathology; Optic nerve diseases/diagnosis; Tomography, optical coherence/methods; Visual pathways; Case reports [publication type]

\section{REFERÊNCIAS}

1. Miller NR, Newmann NJ. Walsh and Hoyt's clinical neuro-ophthalmology. 5th ed. Baltimore: Williams \& Wilkins; 1998. p.336.

2. Luco C, Hoppe A, Schweitzer M, Vicuña X, Fantin A. Visual field defects in vascular lesions of the lateral geniculate body. J Neurol Neurosurg Psychiat. 1992;55(1):12-5.

3. Monteiro ML, Leal BC, Rosa AA, Bronstein MD. Optical coherence tomography analysis of axonal loss in band atrophy of the optic nerve. $\mathrm{Br} \mathrm{J}$ Ophthalmol. 2004;88(7):896-9.

4. Newman SA, Miller NR. Optic tract syndrome. Neuro-ophthalmologic considerations. Arch Ophthalmol. 1983;101(8):1241-50.

5. Gunderson $\mathrm{CH}$, Hoyt WF. Geniculate hemianopia: incongruous homonymous field defects in two patients with partial lesions of the lateral geniculate nucleus. J Neurol Neurosurg Psychiatry. 1971;34(1):1-6.

6. Frisén L, Holmegaard L, Rosencrantz M. Sectorial optic atrophy and homonymous, horizontal sectoranopia: a lateral choroidal artery syndrome? J Neurol Neurosurg Psychiatry. 1978;41(4):374-80.

7. Helgason C, Caplan LR, Goodwin J, Hedges T 3rd. Anterior choroidal arteryterritory infarction. Report of cases and review. Arch Neurol. 1986;43(7):681-6.

8. Donahue SP, Kardon RH, Thompson HS. Hourglass-shaped visual fields as a sign of bilateral lateral geniculate myelinolysis. Am J Ophthalmol. 1995;119(3): 378-80.

9. Grochowicki M, Vighetto A. Homonymous horizontal sectoranopia: report of four cases. Br J Ophthalmol. 1991;75(10):624-8.

10. Shacklett DE, O'Connor PS, Dorwart RH, Linn D, Carter JE. Congruous and incongruous sectoral visual field defects with lesions of the lateral geniculate nucleus. Am J Ophthalmol. 1984;98(3):283-90.

11. Borruat FX, Maeder P. Sectoranopia after head trauma: evidence of lateral geniculate body lesion on MRI. Neurology. 1995;45(3 Pt 1):590-2.

12. Horton JC, Landau K, Maeder P, Hoyt WF. Magnetic resonance imaging of the human lateral geniculate body. Arch Neurol. 1990;47(11):1201-6. 